\title{
Results from the Telescope Array experiment
}

\author{
Hisao Tokuno* for The Telescope Array Collaboration \\ Department of Physics \\ Tokyo Institute of Technology \\ Meguro, Tokyo, Japan \\ E-mail: htokunodcr.phys.titech.ac.jp
}

Since May 2008, the Telescope Array (TA) experiment has been observing ultra-high energy cosmic rays (UHECRs) to study their origins. The TA is a hybrid detector consisting of a surface detector (SD) array and air fluorescence detectors (FDs) at three stations surrounding the SD array. We present our recent results of the energy spectrum, mass composition, and arrival direction of UHECRs. The energy spectrum from the SD shows a cutoff structure at $48 \mathrm{EeV}$ with $3.9 \sigma$ from a linear extrapolation of the energy spectrum below the cutoff energy. The preliminary results of a mass composition study with stereo FD analysis shows that a proton primary is dominant above $10^{18.2} \mathrm{eV}$. Our arrival direction studies of UHECRs do not indicate significant anistropy.

36th International Conference on High Energy Physics,

July 4-11, 2012

Melbourne, Australia

${ }^{*}$ Speaker. 


\section{Introduction}

The Telescope Array (TA) experiment [ $[$ ] has been observing ultra-high energy cosmic rays (UHECRs) an aim of understanding their origins. The TA is a hybrid detector consisting of a surface detector (SD) array and fluorescence detectors (FDs) at three stations surrounding the SD array. The commissioning run of the hybrid detector ended in May 2008, and the hybrid detector has been running continuously since then. In our analysis, we can select a combination of detectors for specific purposes: monocular FD, stereo FD, FD with one SD, and SD only. Analysis results from these combinations are compared with each other in order to verify the self-consistency of the detector.

Five hundred and seven SDs are arrayed on a grid with $1.2 \mathrm{~km}$ spacing, and the area of the array is approximately $700 \mathrm{~km}^{2}$. The arrival direction and the energy of the primary cosmic ray is reconstructed from the arrival timings and the energy deposits of the extensive air shower (EAS) particles. SD performance parameters, such as PMT gain and trigger rate are monitored every $10 \mathrm{~min}$, and monitored data are stored on a central data acquisition (DAQ) system. Ninety-five percent of the SDs can be used for the analysis, and the duty cycle of the SD array is 98\%, which includes the daily and yearly maintenance periods [ [2]. The reconstruction efficiency (including trigger efficiency) is $10 \%$ with energies at $10^{18.2} \mathrm{eV}$, and the efficiency rises up to $100 \%$ above $10^{18.9} \mathrm{eV}[$ [B]].

The longitudinal developmen of EAS is observed by using the FDs to measure the energy and particle type of the primary cosmic ray. Three FD stations are located at the black rock mesa (BRM), the Long Ridge (LR), and the Middle Drum (MD) [ [⿴囗十, 目, 团]. The FDs on the BRM and LR station were newly constructed for the TA experiment, and the FDs on the MD station were moved from the HiRes-I experiment. FD performance parameters, such as PMT gain, mirror reflectance and window transmittance, are monitored continuously. The duty factor of FD is $\sim 9 \%$.

We compared the energy scale of both the FDs and the SD array, and found that the energy scale of SD is $27 \%$ higher than that of FD. In the SD analysis, we obtain the energy deposit at $800 \mathrm{~m}$ from the EAS core with a lateral distribution function, and compare it with the simulated results in order to estimate primary energy. This energy determination method depends on the accuracies of the extrapolations of a hadronic interaction model from the confirmed energy regions by accelerator measurements to the UHE regions. Conversely, the FD primary energy measurement is calorimetric in principle, and its systematic uncertainties can be investigated by performing experimental measurements. Therefore, the SD energy scale is shifted by $1 / 1.27$ in order to equal the FD energy scale. The left panel in Figure $\square$ shows a comparison of the reconstructed energy between FD and SD (scaled) for the same events. We consider systematic uncertainties of energy detemination of $\pm 22 \%$ obtained from the uncertainties of FD energy determination [[], 团].

\section{Results}

\subsection{Energy Spectrum}

The SD array has been running continuously since May 2008, and the total exposure reached approximately 2640 [ $\mathrm{km}^{2} \mathrm{sr} \mathrm{yr}$ ] in April 2011 [B]]. The energy spectrum above $10^{18.2} \mathrm{eV}$ obtained

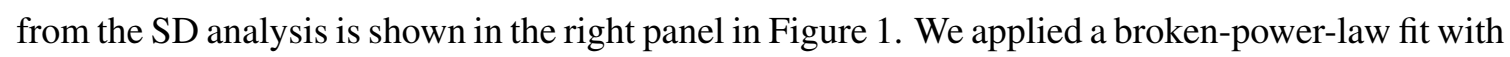



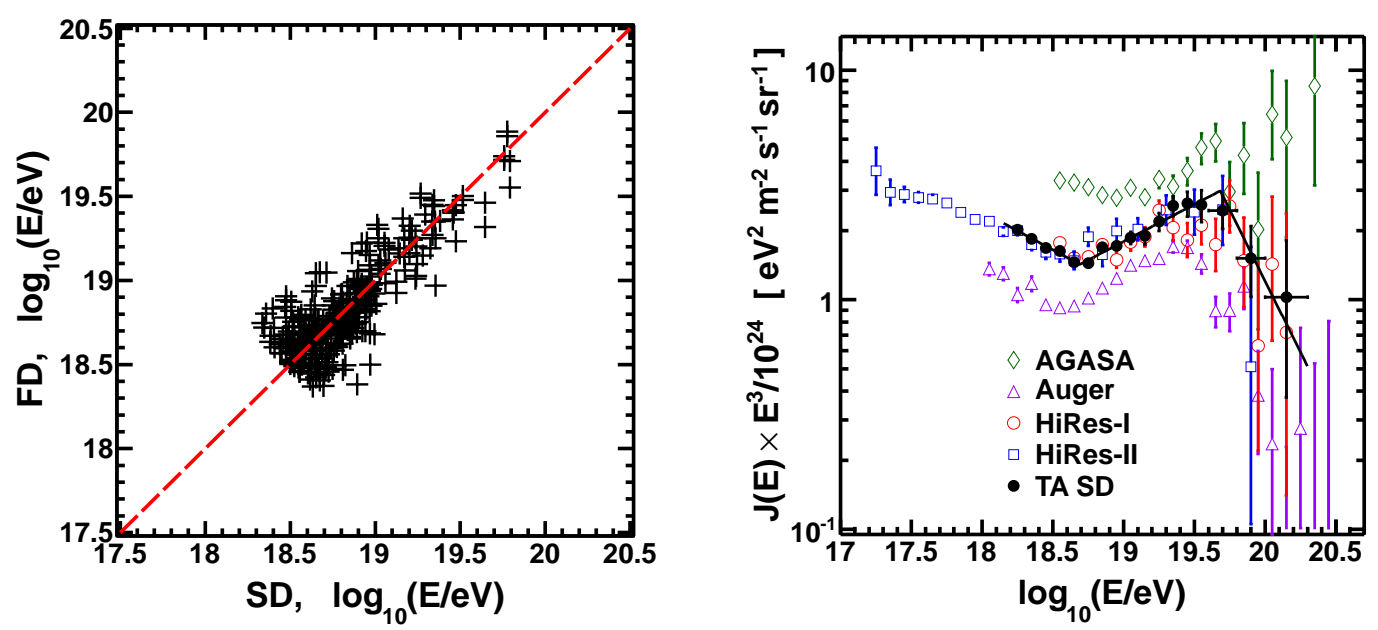

Figure 1: Left panel: Comparison between the energy obtained from FD (hybrid events) and shifted SD $(1 / 1.27)$ for the same events. Right panel: Energy spectrum of UHECRs measured by the SD array (filled circles).

two bending points to the SD spectrum. The best fit value of the two bending points were obtained at $E=4.9 \times 10^{18} \mathrm{eV}$ and $4.8 \times 10^{19} \mathrm{eV}$. The number of events observed above the cut-off point is 28 , and the expected number of events with an assumption of linear extrapolation below the cut-off energy is 54.9. The significance of the cut-off was evaluated from the Poisson probability as $3.9 \sigma$. For comparison, spectra from AGASA, HiRes-I, HiRes-II, and the Pierre Auger Observatory are also shown in this figure. Our spectrum is good agreement with the HiRes-I and HiRes-II results, and our spectrum is also consistent with the other results, with systematic uncertainties of $\sim 20 \%$ for the primary energy determination.

\subsection{Composition}

The longitudinal development of EAS strongly depends on the primary particle type and energy, and the maximal development point of $\operatorname{EAS}\left(X_{\max }\left[\mathrm{g} \mathrm{cm}^{-2}\right]\right)$ is sensitive to the primary particle type. To study the primary particle type, we compared the observed $X_{\max }$ distributions with the simulated ones in several energy ranges [ [ $]$ ]. The event simulation included the detector responses, and atmospheric conditions in the simulations were the same as those during the real events. The simulated events are reconstructed using the same event reconstruction program for the real observed events, and the same data selection criteria are applied for the observed and simulated events.

Figure $\square$ shows the observed $X_{\max }$ distributions analyzed by the FD stereo measurement, and the simulated $X_{\max }$ distributions for the proton and iron primary in different energy ranges. In this figure, the QGSJET-II hadronic interaction model is used for simulating the $X_{\max }$ distributions. The distributions of $X_{\max }$ are consistent with the simulated distribution having proton primary below the energy of $10^{19.4} \mathrm{eV}$. It is difficult to determine the dominant particle type in the highest energy range of $10^{19.4-19.6} \mathrm{eV}$ because of low statistics. We are presently studying systematic uncertainties of this measurement and the contribution from other light components such as helium. 

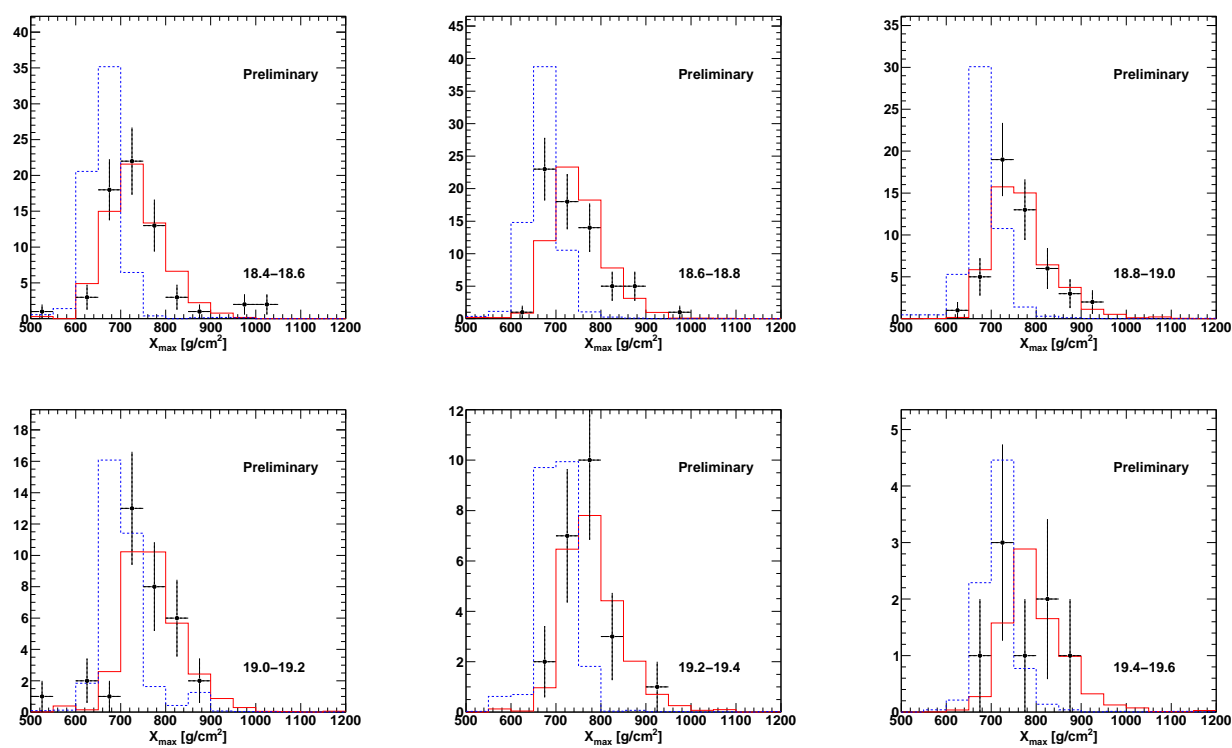

Figure 2: The preliminary result of the $X_{\max }$ distributions reconstructed by the stereo FD analysis with the different energy ranges. Plots: the observed $X_{\max }$. Histograms: the simulated $X_{\max }$ distribution for proton (histogram with solid line) and iron (histogram with broken line) with the hadronic interaction model of QGSJET-II. The simulated $\mathrm{X}_{\max }$ includes detector responses of the $\mathrm{FD}$, and the reconstruction biases same as measured one.

\subsection{Anisotropy}

Anisotropy in the arrival direction of UHECR provides important information about the origin of UHECRs. We investigated the auto-correlation of the arrival directions themselves [Q]]. The number of pairs in a given window size is counted, and its chance probability from an isotropic distribution is calculated. From this analysis, we obtained relatively smaller chance probabilities from the observed event set with energies higher than $57 \mathrm{EeV}$ and window sizes of $\sim 15^{\circ}$; however, these are not significant.

The Pierre Auger Observatory (PAO) has reported that the arrival directions of UHECR events with energies of $E>57 \mathrm{EeV}$ are correlated with active galactic nuclei (AGN) in the object catalog (the Veron-Cetty and Veron (VCV) catalog [प]]) with redshift smaller than 0.018 . The best correlation was observed at a search window of $3.1^{\circ}$ from the arrival directions of UHECRs [ए], [2], [3]]. We studied the same correlation for our observed events with the same parameter set [Q]]. The left panel in Figure B shows the observed arrival direction of CR with the same parameter set. The number of observed events that correlate the AGNs is 11 out of 25 , and the number of expected chance correlation is 5.9 from an isotropic arrival direction. This excess has a chance probability of $\sim 2 \%$, estimated using the binomial distribution with a probability of a single isotropic event to correlate the AGNs. Therefore, we can conclude that the present TA data indicated no significant correlation with the AGNs claimed by PAO.

We investigated a correlation between the arrival directions of UHECRs and the large scale structure (LSS) of matter distribution [Q]. The expected cosmic ray arrival direction is calculated 

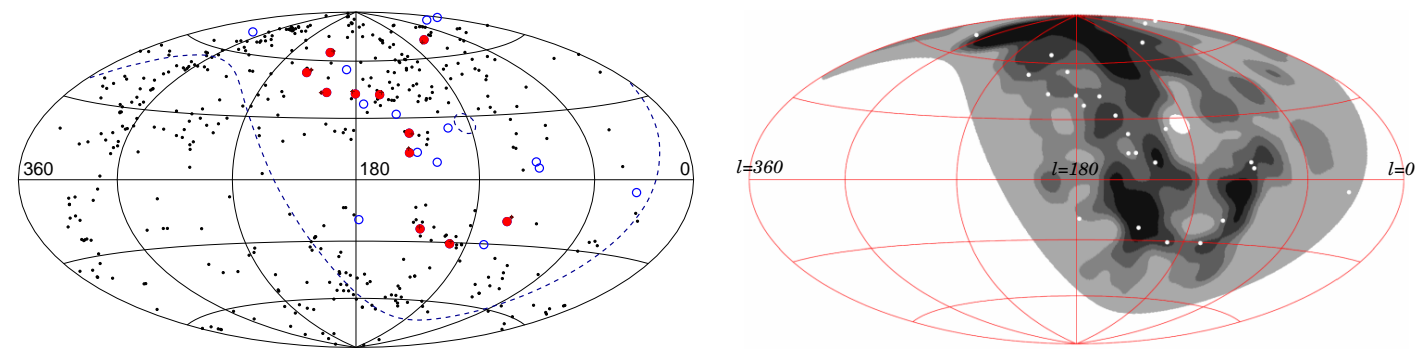

Figure 3: The arrival direction of observed UHECR with primary energy of $E>57 \mathrm{EeV}$ in galactic coordinates. In the left panel, dots represent AGN with the $z<0.018$, filled circles indicate the correlated arrival direction with the AGN within $3.1^{\circ}$, open circles represent the uncorrelated cosmic rays. In the right panel, white dots represent the arrival directions of observed UHECRs with primary energy of $E>57 \mathrm{EeV}$, and the contour indicates a flux density predicted using a LSS model with a smear angle of $6^{\circ}$.

by using the flux sampling method [14] with objects at distances shorter than $250 \mathrm{Mpc}$ from the preliminary version of 2MASS Galaxy Redshift Catalog [피]. In the calculation, we considered the following aspects; smearing angles by the galactic and extra-galactic magnetic fields, the isotropic contribution of sources far from the $250 \mathrm{Mpc}$ attenuation due to cosmic ray interactions, and TA exposure. The right panel in Figure 3 shows the observed arrival directions of the UHECRs and a flux density predicted from the LSS model with a smearing angle of $6^{\circ}$. From these comparisons, the present TA data indicates no significant difference between the distribution obtained according to LSS model and the isotropic distribution.

\section{New equipment}

To reduce the systematic uncertainty of the FD energy determination, we are measuring the fluorescence lights from controlled electrons in the air using our FDs [ए6]. The electron light source (ELS) module shoots a $10^{9}$ electron bunch with an energy of $40 \mathrm{MeV}$. This ELS functions as a standard candle of fluorescence light. This module has been installed at the front of the BRM station at a distance of $100 \mathrm{~m}$ from the FDs. The fluorescence light measurement enables us an end-to-end calibration that includes the fluorescence yields and the performance of our FDs.

To precisely measure the energy spectrum and mass composition of cosmic rays with energies around $10^{17}-10^{18} \mathrm{eV}$, we are constructing a new FD building in the MD station. This project is called as The TA Low-energy Extension (TALE) [ []]. The FDs used at the HiRes-II experiment will be installed in the new building, and their FOV are overlapped with and more higher than that of the existing FDs at the MD station. The FOV of the new and the existing FDs covers an elevation angle from $3^{\circ}$ to $59^{\circ}$, and this larger angle enables the measurement of the $X_{\max }$ of lower energy cosmic rays with a smaller bias. For precisely measuring EAS, we are also installing an infill SD array between the MD station and the TA-SD array. This infill array consists of two sub-arrays with different spacings of $400 \mathrm{~m}$ and $600 \mathrm{~m}$.

\section{Acknowledgments}

The Telescope Array experiment is supported by the Japan Society for the Promotion of Sci- 
ence through Grants-in-Aid for Scientific Research on Specially Promoted Research (21000002) "Extreme Phenomena in the Universe Explored by Highest Energy Cosmic Rays", and the InterUniversity Research Program of the Institute for Cosmic Ray Research; by the U.S. National Science Foundation awards PHY-0307098, PHY-0601915, PHY-0703893, PHY-0758342, and PHY0848320 (Utah) and PHY-0649681 (Rutgers); by the National Research Foundation of Korea (2006-0050031, 2007-0056005, 2007-0093860, 2010-0011378, 2010-0028071, R32-10130); and by the Russian Academy of Sciences, RFBR grants 10-02-01406a and 11-02-01528a (INR), IISN project No. 4.4509.10 and Belgian Science Policy under IUAP VI/11 (ULB). The foundations of Dr. Ezekiel R. and Edna Wattis Dumke, Willard L. Eccles, and the George S. and Dolores Dore Eccles all helped with generous donations. The State of Utah supported the project through its Economic Development Board, and the University of Utah through the Office of the Vice President for Research. The experimental site became available through the cooperation of the Utah School and Institutional Trust Lands Administration (SITLA), U.S. Bureau of Land Management, and the U.S. Air Force. We also wish to thank the people and the officials of Millard County, Utah, for their steadfast and warm support. We gratefully acknowledge the contributions from the technical staff of our home institutions and the University of Utah Center for High Performance Computing (CHPC).

\section{References}

[1] H. Kawai, et al., Nucl. Phys. B Proc. Suppl, 175-176 (2008) 221

[2] T. Abu-Zayyad, et al., Nucl. Instr. and Meth. A, 689 (2012) 87

[3] T. Abu-Zayyad, et al., arXiv:1205.5067.v1

[4] H. Tokuno, et al., Nucl. Instr. and Meth. A, 676 (2012) 54

[5] J.N. Matthews, et al., Proceedings of 30th International Cosmic Ray Conference, 5 (2007) 1157

[6] T. Abu-Zayyad, et al. Astropart. Phys, (2012), in Press; arXiv:1202.5141

[7] T. Abu-Zayyad, et al., Proceedings of 32nd International Cosmic Ray Conference, 2 (2011) 250

[8] Y. Tameda, et al., AIP Conf. Proc., 1367 (2011) 110

[9] T. Abu-Zayyad, et al. ApJ, 757 (2012) 26

[10] M.P. Veron-Cetty and P. Veron, Astrophys. 455 (2006) 773

[11] P. Abreu, et al., Science, 318 (2007) 938

[12] P. Abreu, et al., Astropart. Phys. 29 (2008) 188

[13] P. Abreu, et al., Astropart. Phys. 34 (2010) 314

[14] H.B.J. Koers and P. Tinyakov, JCAP, 0904 (2009) 003; Mon. Not. Roy. Astron. Soc., 399 (2009) 1005

[15] T. Jarrett, Personal communication

[16] T. Shibata, et al., Nucl. Instr. and Meth. A, 597 (2008) 61 